\title{
Sistema de recirculação de água com reator aerado em membrana na produção intensiva de tilápia
}

Water recirculation system with aerial membrane reactor in intensive tilapy production

Sistema de recirculación de agua con reactor de membrana aérea en la producción intensiva de tilapia

Juliana Cristina Ribeiro Almeida Mestranda em Recursos Hídricos e Tecnologias Ambientais, bolsista CAPES, UNESP, Brasil. juliana-almeida15@hotmail.com

João Miguel Merces Bega Mestrando em Recursos Hídricos e Tecnologias Ambientais, bolsista CNPq, UNESP, Brasil. joaomiguelbega@gmail.com

\section{Natasha Ulhiana Ferreira Ribeiro} Mestranda em Recursos Hídricos e Tecnologias Ambientais, bolsista CAPES, UNESP, Brasil. natasha_ul@yahoo.com.br

Amanda de Moraes Ricardi Mestranda em Recursos Hídricos e Tecnologias Ambientais, bolsista CNPq, UNESP, Brasil. amandademricardi@gmail.com

Tsunao Matsumoto Professor Doutor, UNESP, Brasil. tsunao.matsumoto@unesp.br 


\section{Fórum Amb enta

\section{RESUMO}

A piscicultura, que é um ramo da aquicultura, tem tido um elevado crescimento no mundo todo, principalmente no Brasil. Dentro das espécies mais cultivas no Brasil estão as Tilápias do Nilo (Oreochromis niloticus). Com esse crescimento, também se aumenta a preocupação na busca por alternativas de criação de peixes. Os Sistemas de Recirculação de Água (SRA) são sistemas de criação fechados e representam alternativas compactas para cultivo intensivo de espécies com elevadas densidades de estocagem. Os SRA têm a vantagem de total controle do efluente e também podem ser instalados perto de grandes centros urbanos. O objetivo central desta pesquisa foi de avaliar a eficiência da transferência de oxigênio dissolvido por um Reator de Biofilme Aerado em Membrana (MABR) e monitorar parâmetros básicos para sobrevivência dos peixes, sendo estes: $\mathrm{pH}$, temperatura e oxigênio dissolvido. O sistema de cultivo foi composto por um tanque com uma densidade de estocagem de $10,12 \mathbf{~ k g} \cdot \mathbf{m}^{-3}$ de tilápias. No interior do MABR foi instalada uma membrana de silicone de fibra oca, que foi suporte para o biofilme. O MABR e a membrana foram aerados por um soprador de ar. Foram realizados ensaios de aeração com recirculação de água com vazões de ar de 50, 100 e $200 \mathbf{~ L . ~ h} \mathbf{h}^{\mathbf{- 1}}$. Os valores encontrados de taxa de transferência de oxigênio para as respectivas vazões foram de 4,92; 7,66 e 7,66 g. $\mathbf{O}_{2} \cdot \mathbf{m}^{-2} \cdot \mathbf{d i a}^{-1}$.

PALAVRAS-CHAVE: Piscicultura. Água residuária. Membrana aerada.

\section{ABSTRACT}

Fish farming, which is a branch of aquaculture, has had a high growth worldwide, especially in Brazil. Among the most cultivated species in Brazil are the Nile Tilapia (Oreochromis niloticus). With this growth, there is also increased concern in the search for fish farming alternatives. Water Recirculation Systems (SARS) are closed rearing systems and represent compact alternatives for intensive cultivation of species with high stocking densities. The SARs have the advantage of full effluent control and can also be installed near large urban centers. The main objective of this research was to evaluate the efficiency of dissolved oxygen transfer by a Membrane Aerated Biofilm Reactor (MABR) and to monitor basic parameters for fish survival, such as $\mathrm{pH}$, temperature and dissolved oxygen. The culture system consisted of a tank with a stocking density of $10.12 \mathrm{~kg} \cdot \mathrm{m}^{-3}$ of tilapia. Inside the MABR was installed a hollow fiber silicone membrane, which was support for the biofilm. The MABR and the membrane were aerated by an air blower. Aeration tests were performed with water recirculation at 50, 100 and $200 \mathbf{L} \cdot \boldsymbol{h}^{-1}$. Oxygen transfer rate values for the respective flow rates were 4.92; 7.66 and 7.66 g. $\mathrm{O}_{2} \cdot \mathrm{m}^{-2} \cdot \mathrm{dia}^{-1}$.

KEYWORDS: Pisciculture. Wastewater. Aerated membrane.

\section{RESUMEN}

La piscicultura, que es una rama de la acuicultura, ha tenido un alto crecimiento en todo el mundo, especialmente en Brasil. Entre las especies más cultivadas en Brasil se encuentran la tilapia del Nilo (Oreochromis niloticus). Con este crecimiento, también existe una mayor preocupación en la búsqueda de alternativas de piscicultura. Los sistemas de recirculación de agua (SARS) son sistemas de cría cerrados y representan alternativas compactas para el cultivo intensivo de especies con altas densidades de población. Los SAR tienen la ventaja de un control total de los efluentes y también se pueden instalar cerca de grandes centros urbanos. El objetivo principal de esta investigación fue evaluar la eficiencia de la transferencia de oxígeno disuelto por un Reactor de Biofilm Aireado de Membrana (MABR) y monitorear parámetros básicos para la supervivencia de los peces, como el $\mathrm{pH}$, la temperatura y el oxígeno disuelto. El sistema de cultivo consistió en un tanque con una densidad de población de $10.12 \mathrm{~kg} \cdot \mathrm{m}^{-3}$ de tilapia. Dentro del MABR se instaló una membrana de silicona de fibra hueca, que era el soporte para la biopelícula. EI MABR y la membrana fueron aireados por un soplador de aire. Las pruebas de aireación se realizaron con recirculación de agua a 50, 100 y $200 \mathbf{L}^{-h^{-1}}$. Los valores de velocidad de transferencia de oxígeno para las velocidades de flujo respectivas fueron 4,92; 7.66 y $7.66 \mathrm{~g} . \mathrm{o}_{\mathbf{2}} \cdot \mathrm{m}^{-2}$. dia $\mathbf{d i}^{\mathbf{1}}$.

PALABRAS CLAVE: Piscicultura. Aguas residuales. Membrana aireada. 


\section{INTRODUÇÃO}

A aquicultura define-se como a atividade de produção de espécies hidrobiológicas de cultivo sob condições controladas ou semicontroladas. A piscicultura, que é um ramo da aquicultura, trata-se da produção especificamente de peixes, que é uma atividade praticada desde a antiguidade (MAIGUEL ENRIQUEZ, 2011).

Tem-se que a produção de pescados é dividida entre a pesca extrativa e a aquicultura. A pesca é a atividade que se baseia na retirada de recursos pesqueiros do ambiente natural, enquanto que a aquicultura é o cultivo, normalmente em um espaço confinado e controlado, de organismos aquáticos ou qualquer outra forma de vida aquática de interesse econômico produtivo. A atividade produtiva se divide em diferentes modalidades: piscicultura (criação de peixes); carcinicultura (criação de camarões); ranicultura (criação de rãs); malacocultura (criação de moluscos, ostras e mexilhões); algicultura (cultivo de algas) e outras espécies com menor apelo comercial, tais como a quelonicultura (criação de tartarugas e tracajás) e a criação de jacarés (SCHULTER, 2017).

A Organização das Nações Unidas para Agricultura e Alimentação (FAO) divulgou o relatório do State of The World Fisheries and Aquaculture (SOFIA, 2018), o qual demonstrou que em 2016 a produção global de pescado chegou a 171 milhões de toneladas, com a aquicultura representando $53 \%$ do total de pescado destinado à alimentação humana, excluindo usos como óleo de peixe e ração animal.

O desenvolvimento de novas técnicas de produção no setor da piscicultura proporciona o maior controle do ambiente aquático, que se traduz em ganhos de produtividade e qualidade no cultivo de vários tipos de animais e plantas aquáticas. Trata-se de uma atividade que proporciona benefícios ambientais relevantes, à medida que pode ser praticada em pequenas áreas, reduzindo, assim, o número de hectares para produção de uma maior quantidade de proteínas, contribuindo, portanto, para a redução da pressão antrópica sobre o meio ambiente (SIQUEIRA, 2017).

Os Sistemas de Recirculação de Água (SRA) são sistemas de criação fechados e representam alternativas compactas para cultivo intensivo de espécies com elevadas densidades de estocagem. No SRA, a água circula no sistema e somente uma pequena parcela é renovada diariamente (MASSER; RAKOCY; LOSORDO, 1999).

O sistema de cultivo com recirculação de água tem sido adotado em diversos países como alternativa ao consumo elevado da água (KUBITZA, 2005). Comparado aos sistemas tradicionais de cultivo de peixes em viveiros, os sistemas de recirculação proporcionam menor consumo de água por quilo de peixe produzido, além de terem a vantagem de produzir um valor mínimo de efluente, tendo como consequência a redução do impacto ambiental (BLANCHETON, 2000).

O problema causado pela criação de peixes em tanques com SRA é a alteração nas características da qualidade da água. A excreção e o acúmulo de ração fazem com que seus atributos qualitativos iniciais sejam alterados ao longo do tempo. Com isso, se faz necessário a 
implantação de um sistema de tratamento para que se mantenha a água em condições ideais para o cultivo da espécie.

Os biorreatores são muito utilizados por promoverem a redução ou a eliminação das grandes cargas carbonáceas e nitrogenadas produzidas pela ração e geradas por processos metabólicos dos animais em confinamento. Dentre os biorreatores, tem-se o Reator de Biofilme Aerado em Membranas (MABR), no qual o biofilme é naturalmente imobilizado em uma membrana permeável a oxigênio, de forma que ocorre a difusão do oxigênio através da membrana para o biofilme, promovendo a oxidação dos poluentes (SYRON; CASEY, 2008).

O objetivo central desta pesquisa foi avaliar a taxa de transferência de oxigênio (TTO) de um MABR, o qual foi utilizado em um SRA com produção intensiva de Tilápia do Nilo (Oreochromis niloticus). Para atingir tal objetivo, foram realizados testes para calcular os valores de TTO em diferentes vazões de ar e posteriormente foram analisados os parâmetros básicos para a sobrevivência dos peixes: oxigênio dissolvido, temperatura e potencial hidrogeniônico (pH).

\section{METODOLOGIA}

O experimento foi dividido em duas etapas. A primeira e a segunda fase tiveram o aparato experimental instalado no Laboratório de Saneamento da Faculdade de Engenharia de Ilha Solteira, UNESP. A primeira etapa consistiu em testes para avaliar a TTO, enquanto que a segunda monitorou alguns parâmetros básicos para a sobrevivência dos peixes com o SRA em funcionamento na criação intensiva de tilápias.

\subsection{Primeira etapa}

O aparato experimental contou com soprador de ar, bomba para recirculação de água, tubulações, rotâmetros de água e de ar, mangueira de ar e um reator de biofilme aerado em membrana. Os ensaios desta fase foram feitos utilizando água limpa da torneira. Na Figura 1 é mostrado um esquema geral do aparato. 


\section{Periódica Eletrôniea}

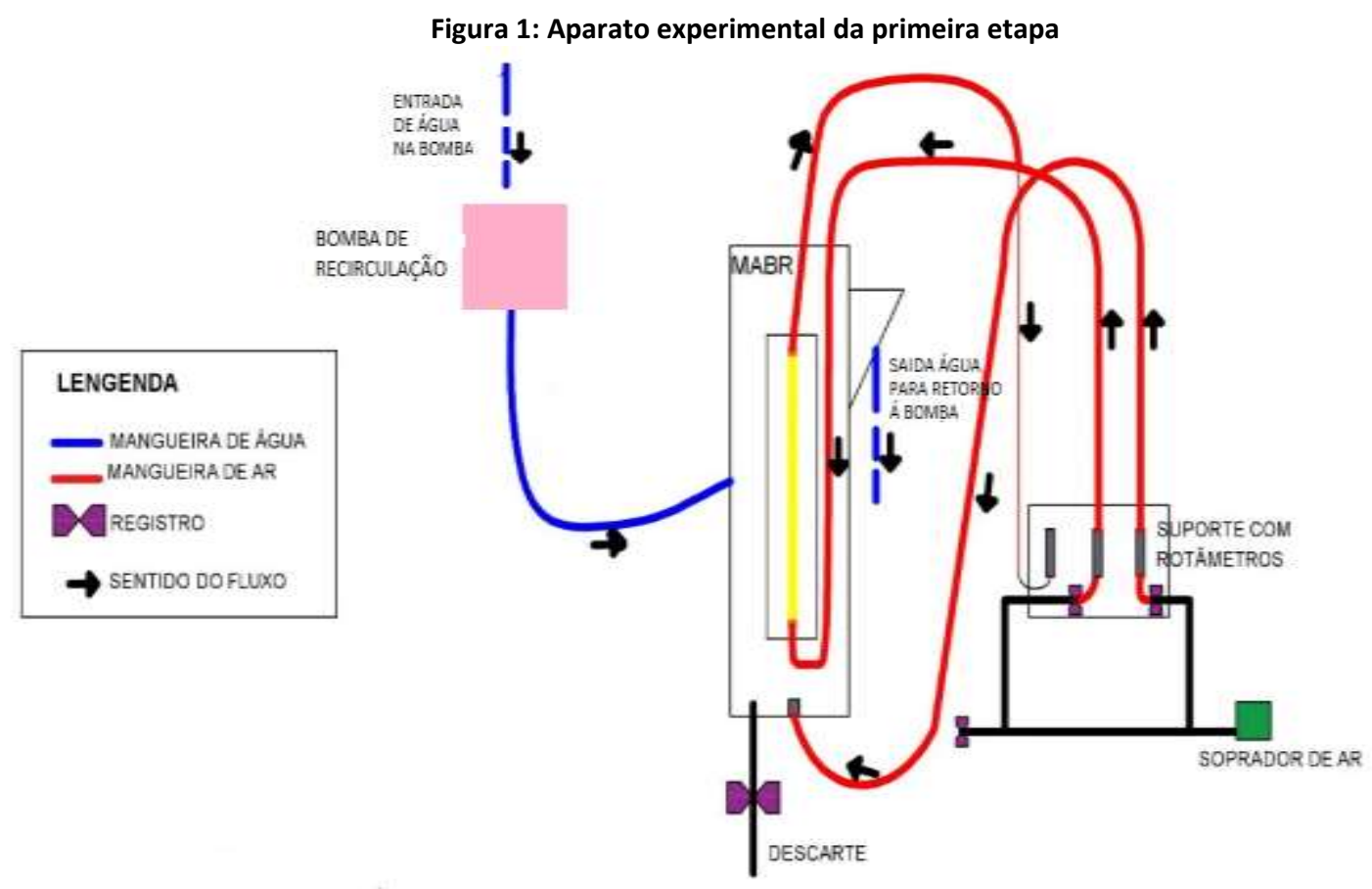

Fonte: Autores.

Para a determinação da contribuição de aeração da membrana para o sistema foram realizados testes variando a vazão de entrada de ar na membrana com vazão constante de recirculação de água de $3,8 \mathrm{~m}^{3} \cdot \mathrm{h}^{-1}$. As vazões de ar aplicadas na membrana de fibra oca foram de 50, 100 e 200 L. s ${ }^{-1}$.

Esta determinação foi estimada a partir do coeficiente global de transferência de oxigênio (KLa), orientada pela metodologia recomendada pela ASCE (1990). A vazão de recirculação de água de $3.8 \mathrm{~m}^{3} \cdot \mathrm{h}^{-1}$ foi baseada nas velocidades mais próximas possíveis dos resultados dos testes de recirculação efetuados por Silva (2017), dentro das possibilidades de atendimento da bomba.

Os ensaios consistiram em encher o reator com água limpa, onde adicionou-se sulfito de sódio como reagente para sequestrar o oxigênio do meio líquido, e cloreto de cobalto, cuja função foi a de catalisar a reação do sulfito. Após a total desoxigenação do meio, iniciou-se a aeração e a recirculação da água limpa até atingir valores próximos ao nível de saturação, de acordo com a temperatura da água e altitude local.

O sulfito de sódio foi adicionado a uma concentração de $7,9 \mathrm{mg}$. $\mathrm{L}^{-1}$ e o cloreto de cobalto a $0,5 \mathrm{mg}$. $\mathrm{L}^{-1}$. Foi então realizada uma recirculação de água no sistema com uma duração de aproximadamente 5 minutos, para que houvesse uma mistura completa e, logo em seguida, o sistema foi mantido em repouso até que o oxigênio dissolvido (OD) se tornasse nulo. Este processo teve a duração de aproximadamente 2 dias.

O monitoramento do OD foi verificado pelo oxímetro da marca YSI, modelo 55-12FT. Assim que os valores se anulavam, iniciava-se o teste com o início da recirculação da água com 3,8 $\mathrm{m}^{3} \cdot \mathrm{h}^{-1}$ de vazão e a vazão de ar da membrana estipulada em cada teste.

No ensaio com as membranas, $O$ OD foi monitorado pelo oxímetro e os valores foram registrados manualmente a cada 5 minutos. O oxímetro foi posicionado dentro do tubo interno, junto com as membranas, centralizado em relação à altura do reator. 
Por fim, o cálculo de KLa foi estimado a partir da Equação 1, onde realizou-se a linearização da curva de OD em função do tempo, obtida no ensaio de aeração. Os valores de KLa foram obtidos através da equação da reta de ajuste, a partir do coeficiente angular da reta.

$$
\ln (\mathrm{Cs}-\mathrm{Ct})=\ln (\mathrm{Cs}-\mathrm{Co})-\left(\mathrm{K}_{\mathrm{La}}\right)^{\mathrm{t}}
$$

onde:

$\mathrm{Cs}=$ concentração de OD na saturação;

$\mathrm{Ct}=$ concentração de OD no tempo t;

Co = concentração de OD inicial;

$\mathrm{t}=$ tempo;

$\mathrm{KLa}=$ coeficiente de transferência de oxigênio $\left(\mathrm{min}^{-1}\right)$.

A taxa de transferência de oxigênio (TTO) é calculada a partir da Equação 2:

$$
\mathrm{TTO}=\mathrm{K}_{\mathrm{La}} \mathrm{x}(\mathrm{Cs}-\mathrm{C}) \times \mathrm{V}
$$

sendo:

$C$ = concentração máxima atingida no ensaio;

$\mathrm{V}=$ volume

\subsection{Segunda etapa}

Nesta fase da pesquisa, o aparato experimental (Figura 2) consistiu em um soprador de ar, bomba para recirculação, tubulações, rotâmetros, mangueira de ar, caixa de nível constante, um decantador de coluna e um reator de biofilme aerado em membrana. O soprador, os rotâmetros e o reator foram os mesmos utilizados na etapa anterior.

Aqui, o sistema foi alimentado com efluente de um SRA e funcionou constantemente. Um tanque com capacidade de 1000 L foi utilizado para criação de tilápias e, no fundo deste, foi instalado uma bomba para captar a água e a direcionar para o sistema. A água captada foi encaminhada para o decantador de coluna e o sobrenadante do decantador foi para a caixa de nível constante. Na caixa, foi direcionado $100 \mathrm{~L} . \mathrm{h}^{-1}$ para o MABR e a vazão excedente retornou ao tanque de cultivo de peixes.

Baseando-se nos testes de recirculação feitos no mesmo reator por Silva (2017), foi estabelecida a vazão de ar de $450 \mathrm{~L} \cdot \mathrm{h}^{-1}$ no difusor, o que gerou uma velocidade de circulação no tubo externo de $6,33 \mathrm{~cm} . \mathrm{s}^{-1}$.

Tem-se então um tanque de cultivo com volume de água de $800 \mathrm{~L}$, mais $400 \mathrm{~L}$ do decantador de coluna, além de $120 \mathrm{~L}$ do MABR, totalizando aproximadamente $1320 \mathrm{~L}$ de volume total no sistema. A vazão de operação do MABR foi de $100 \mathrm{~L} \cdot \mathrm{h}^{-1}$, portanto, concluiu-se que o sistema leva aproximadamente 13 horas para tratar todo o volume, que resulta em quase duas renovações completas de água durante o dia. 
Figura 2: Aparato experimental segunda etapa

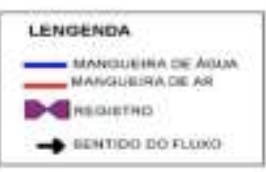

ros
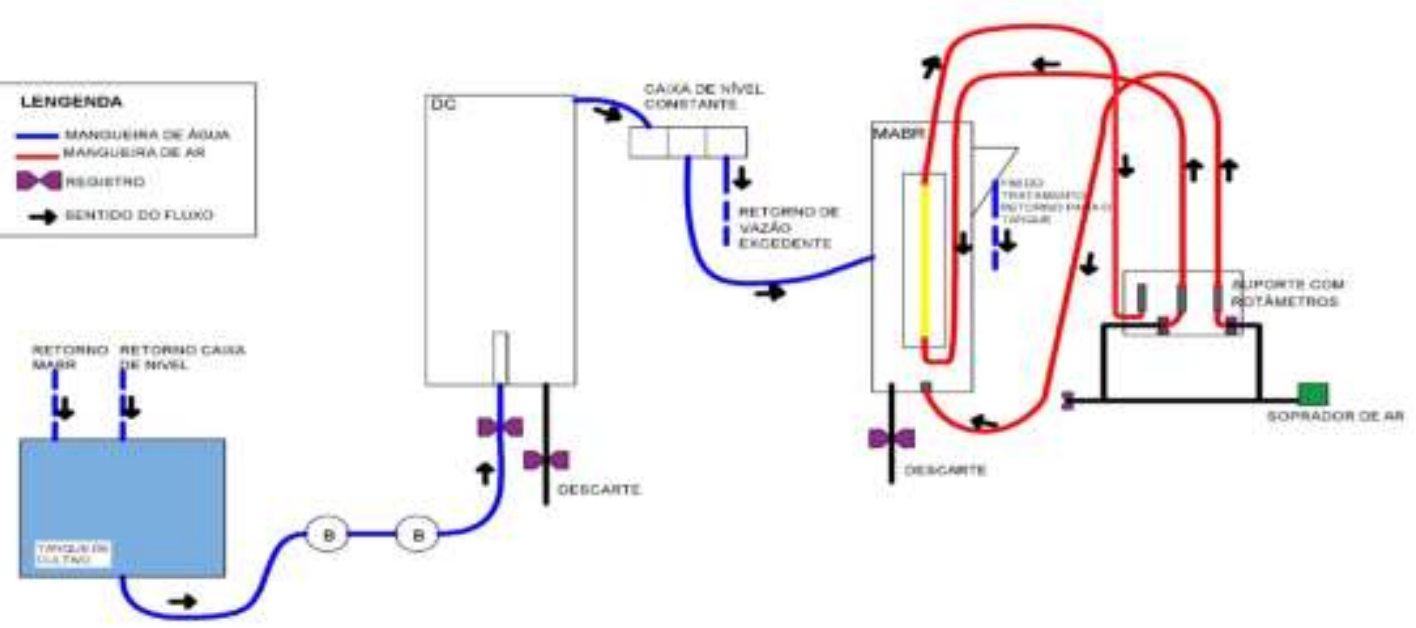

Fonte: Autores.

O MABR foi operado com uma vazão de $100 \mathrm{~L} \cdot \mathrm{h}^{-1}$, mas o decantador de coluna recebia uma vazão total de $400 \mathrm{~L} . \mathrm{h}^{-1}$, o que se deve à algumas limitações do sistema em virtude do conjunto de bombas. Este controle de vazão para o MABR foi feito por meio de uma caixa de nível constante instalada na saída do decantador (DC). A caixa direcionava os $100 \mathrm{~L} \cdot \mathrm{h}^{-1}$ para o reator e o excedente voltava para o tanque de cultivo.

Para monitorar a qualidade da água em relação a alguns parâmetros básicos para os peixes, foram analisados valores de temperatura e $\mathrm{pH}$ medidos dentro do tanque. $\mathrm{O}$ oxigênio dissolvido foi medido em três pontos de amostragem: saída do tanque de cultivo (ST), saída do decantador de coluna (SD) e saída do reator de biofilme aerado em membrana (SR). No Quadro 1 são apresentadas as frequências de amostragens e o método utilizado para determinação dos valores.

\begin{tabular}{|c|c|c|}
\hline \multicolumn{2}{|c|}{ Quadro 1: Parâmetros e frequência das análises e os respectivos métodos utilizados } \\
\hline Parâmetros & Frequência da Analise & Método \\
\hline Oxigênio Dissolvido & Diária & $\begin{array}{c}\text { Medidor de Oxigênio Dissolvido Digital Portátil - } \\
\text { Modelo: MO-900 - Marca: Instrutrerm }\end{array}$ \\
\hline Temperatura & Diária & $\begin{array}{c}\text { Phmetro portátil digital - Modelo: Sdadd - } \\
\text { Marca: Hanna instruments }\end{array}$ \\
\hline pH & Diária &
\end{tabular}

Fonte: Autores.

\section{RESULTADOS E DISCUSSÃO}

\subsection{Primeira etapa}

A solubilidade de oxigênio na água varia de acordo com a temperatura, a altitude e a salinidade (VON SPERLING, 1999). O município de Ilha Solteira, inserido no interior do estado de São Paulo, está localizado a $337 \mathrm{~m}$ de altitude. A temperatura média da água nos ensaios foi de $29^{\circ} \mathrm{C}$, e a salinidade da água foi considerada nula. Assim, a concentração de saturação do oxigênio correspondeu a $7,5 \mathrm{mg}$. $\mathrm{L}^{-1}$. 
Nas Figuras 3, 4 e 5 estão demonstrados os valores de oxigênio dissolvido ao longo do tempo obtidos nos ensaios de aeração das membranas com vazões de 50, 100 e $200 \mathrm{~L} . \mathrm{h}^{-1}$, tendo a recirculação da água controlada pela vazão constante de $3,8 \mathrm{~m}^{3} \cdot \mathrm{h}^{-1}$. Com base nas curvas de oxigenação, aplicou-se a Equação 1. A partir da inclinação da reta de melhor ajuste, obteve-se o KLa. Os gráficos encontrados e as respectivas equações da reta de ajuste estão apresentados nas Figuras 6, 7 e 8.

A Tabela 1 apresenta os valores de KLa obtidos a partir do coeficiente angular das retas da linearização da curva de oxigênio, e também as TTO para o meio líquido com as diferentes vazões de ar. Para o cálculo da TTO, a temperatura média da água utilizada foi de $29^{\circ} \mathrm{C}$ e considerou-se que as 550 membranas contidas no sistema possuíam um total de $1,9 \mathrm{~m}^{2}$ de área superficial.

Tabela 1: Valores dos coeficientes KLa e TTO

\begin{tabular}{|c|c|c|c|}
\hline Ensaio & KLa $\left(\min ^{-1}\right)$ & $\operatorname{KLa}\left(\mathrm{dia}^{-1}\right)$ & $\operatorname{TTO}\left(\mathrm{g} \cdot \mathrm{O}_{2} \cdot \mathrm{m}^{-2} \cdot \mathrm{dia}^{-1}\right)$ \\
\hline 50 L. h $\mathbf{h}^{-1}$ & 0,0003 & 0,432 & 4,9248 \\
\hline $100 \mathbf{L} \cdot \mathbf{h}^{-1}$ & 0,0005 & 0,72 & 7,6608 \\
\hline $200 \mathbf{L} \cdot \mathbf{h}^{-1}$ & 0,0007 & 1,008 & 7,6608 \\
\hline
\end{tabular}

Fonte: Autores.

Figura 3: Curva de oxigenação pela membrana com vazão de ar de 50 L. $\mathbf{s}^{-1}$

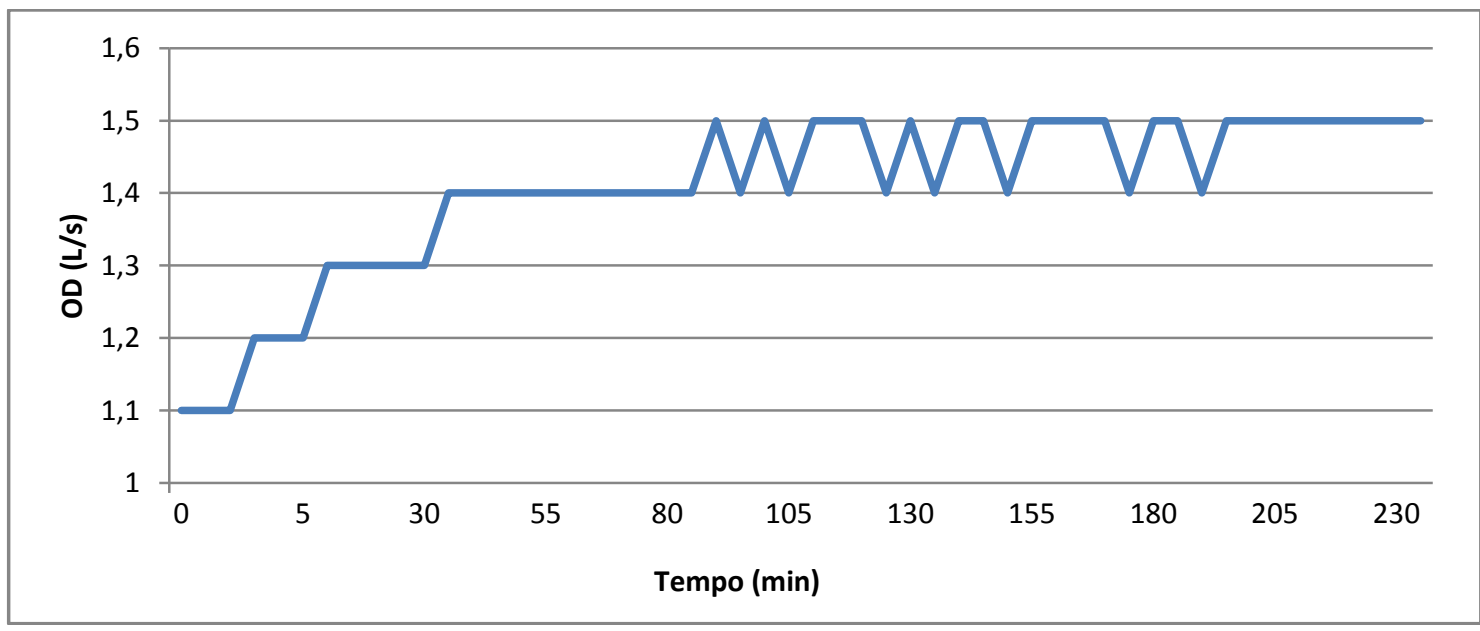

Fonte: Autores. 
Figura 4: Curva de oxigenação pela membrana com vazão de ar de 100 L. $\mathbf{s}^{-1}$

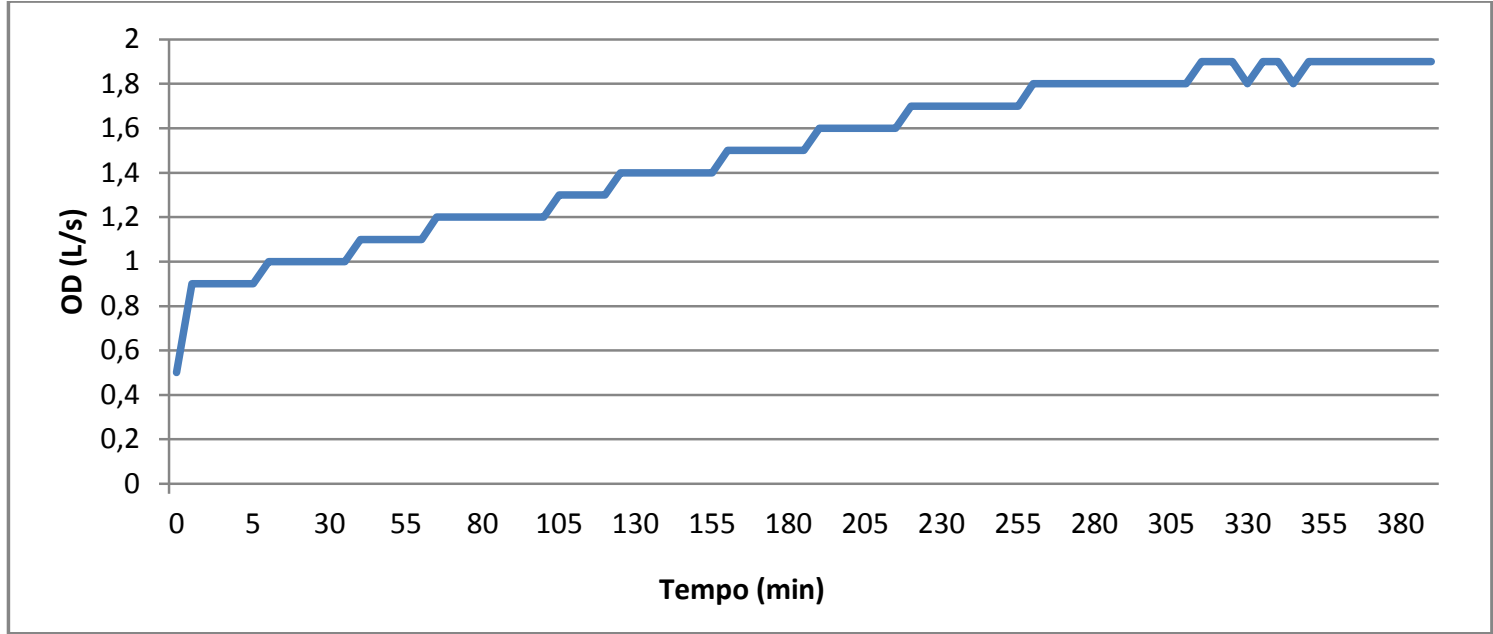

Fonte: Autores.

Figura 5: Curva de oxigenação pela membrana com vazão de ar de 200 L. $\mathbf{s}^{-1}$

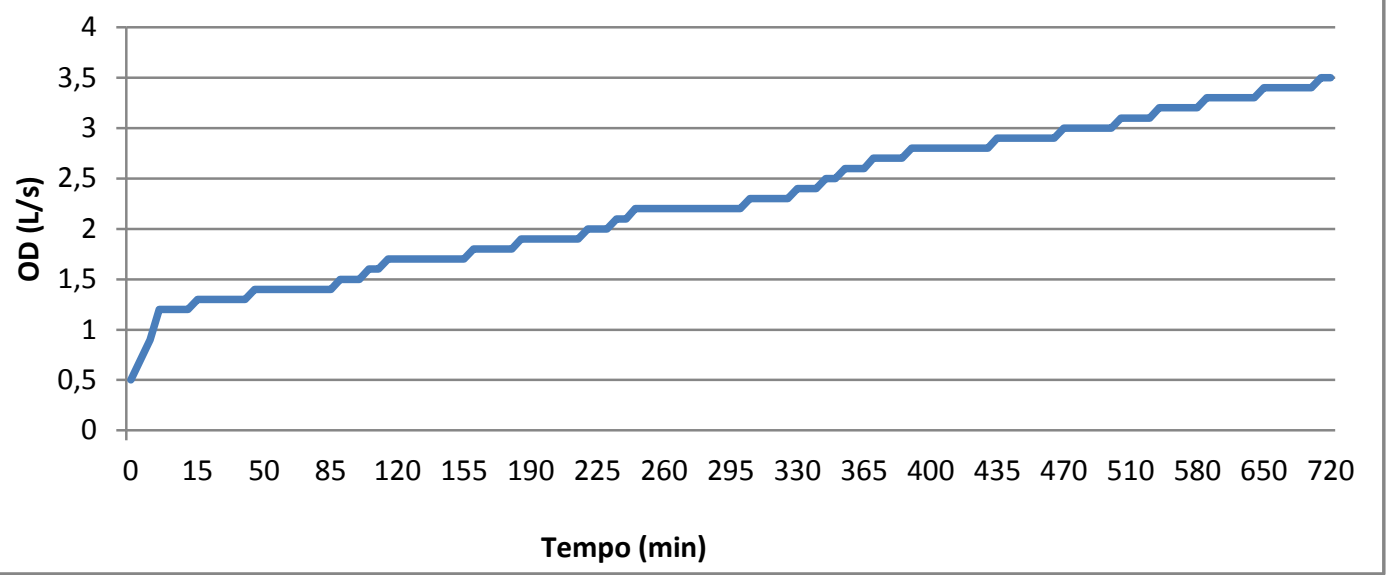

Fonte: Autores.

Figura 6: Linearização da curva de oxigenação com vazão de ar de 50 L. $\mathbf{s}^{-1}$

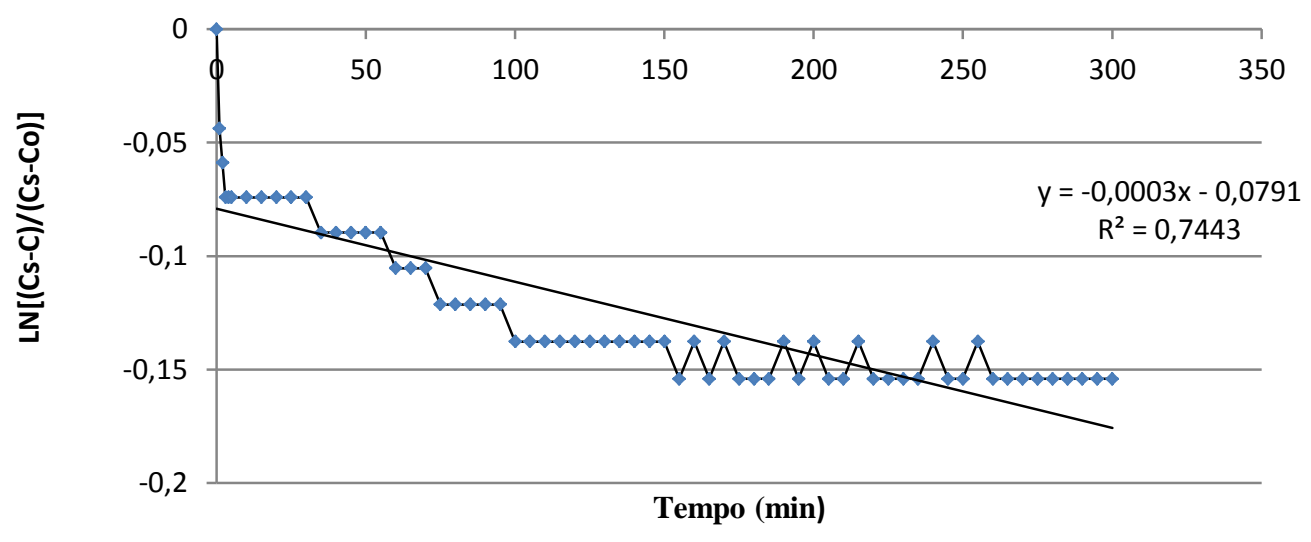

Fonte: Autores. 
Figura 7: Linearização da curva de oxigenação com vazão de ar de 100 L. $\mathbf{s}^{-1}$

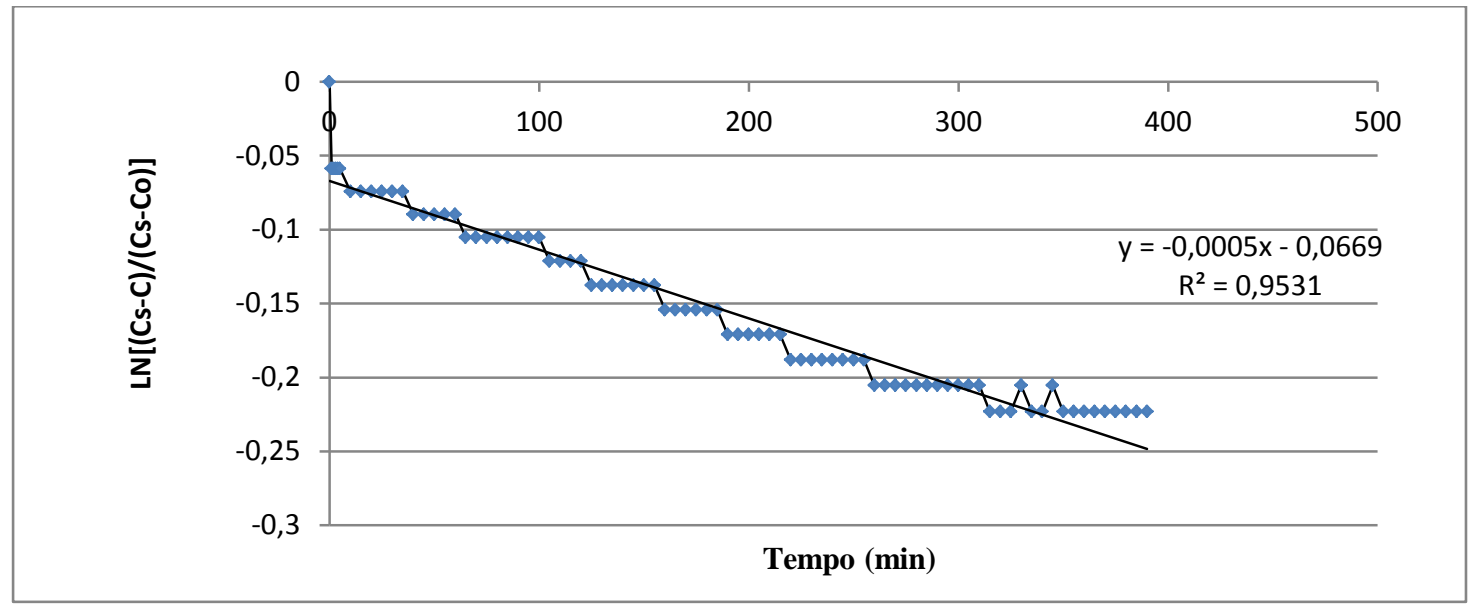

Fonte: Autores.

Figura 8: Linearização da curva de oxigenação com vazão de ar de 200 L. $\mathbf{s}^{-1}$

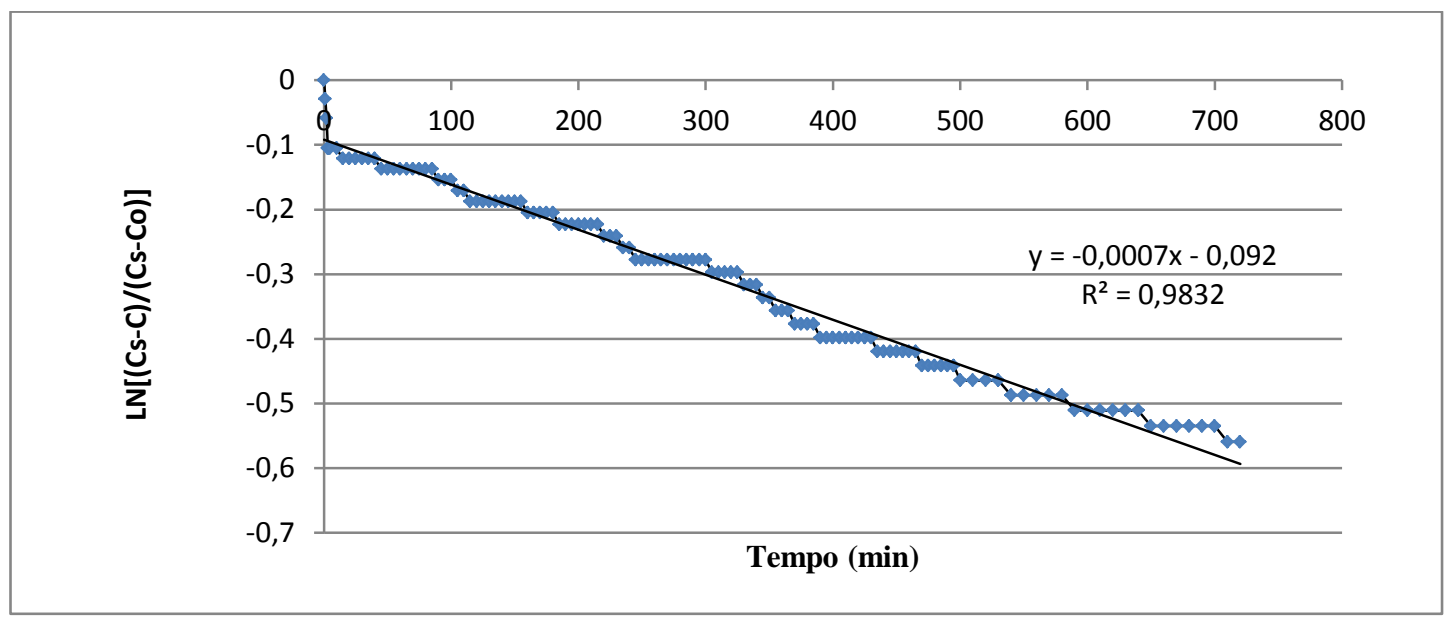

Fonte: Autores.

\subsection{Segunda etapa}

Os resultados dos parâmetros temperatura, pH e oxigênio dissolvido apresentados são de 52 dias de duração do SRA com a criação intensiva de tilápias.

\subsubsection{Temperatura}

Os valores de temperatura medidos dentro do tanque de cultivo estão demonstrados na Figura 9. A temperatura apresentou valor médio de $24,27^{\circ} \mathrm{C}$, com valor mínimo de $19,4^{\circ} \mathrm{e}$ máximo de $26,5^{\circ} \mathrm{C}$. 
Figura 9: Valores de temperatura no tanque nos 52 dias

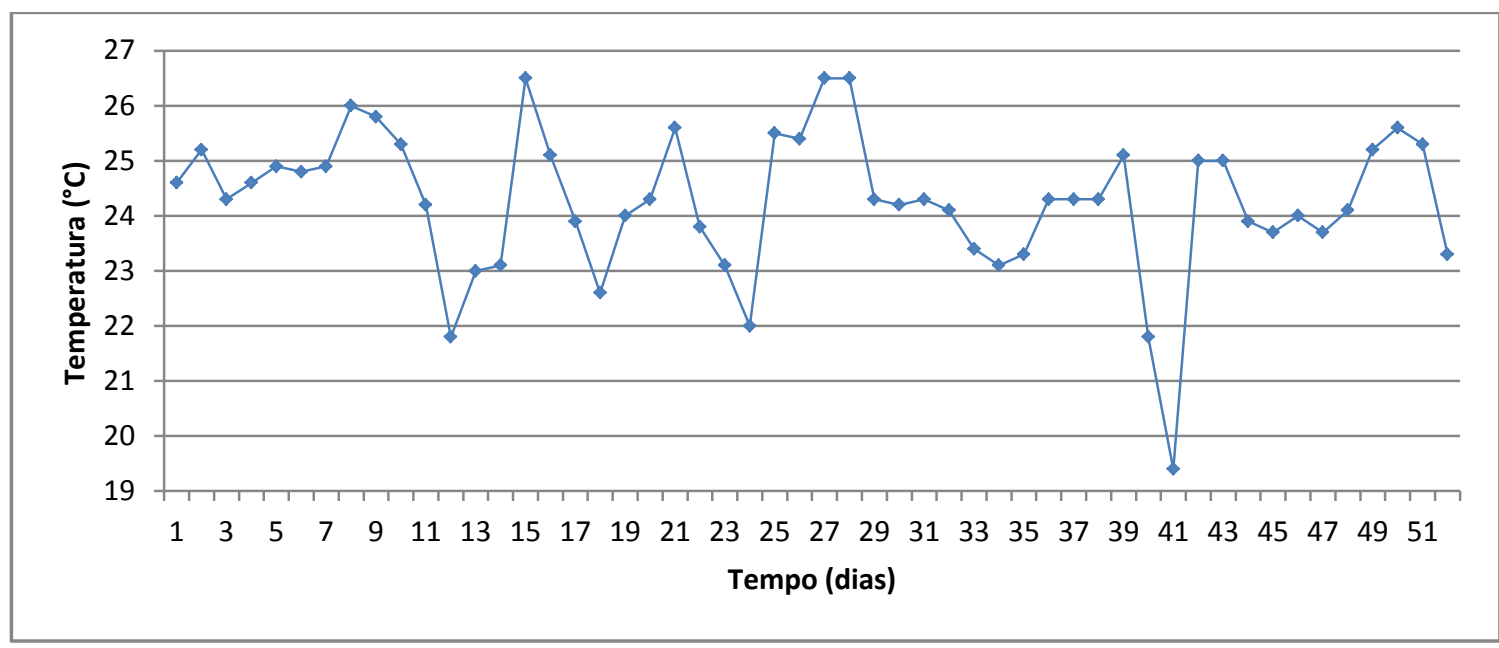

Fonte: Autores.

\subsubsection{Potencial Hidrogeniônico}

Os valores de $\mathrm{pH}$ obtidos no tanque de cultivo estão mostrados na Figura 10. O valor médio de $\mathrm{pH}$ encontrado foi de 7,72, tendo sido verificado os valores mínimo e máximo de 7,0 e 8,3, respectivamente. No geral, o pH teve uma oscilação baixa, sendo mantido uma faixa dentro do recomendado para o cultivo de tilápias segundo Kubtiza (2000).

Figura 10: Valores de pH no tanque nos 52 dias

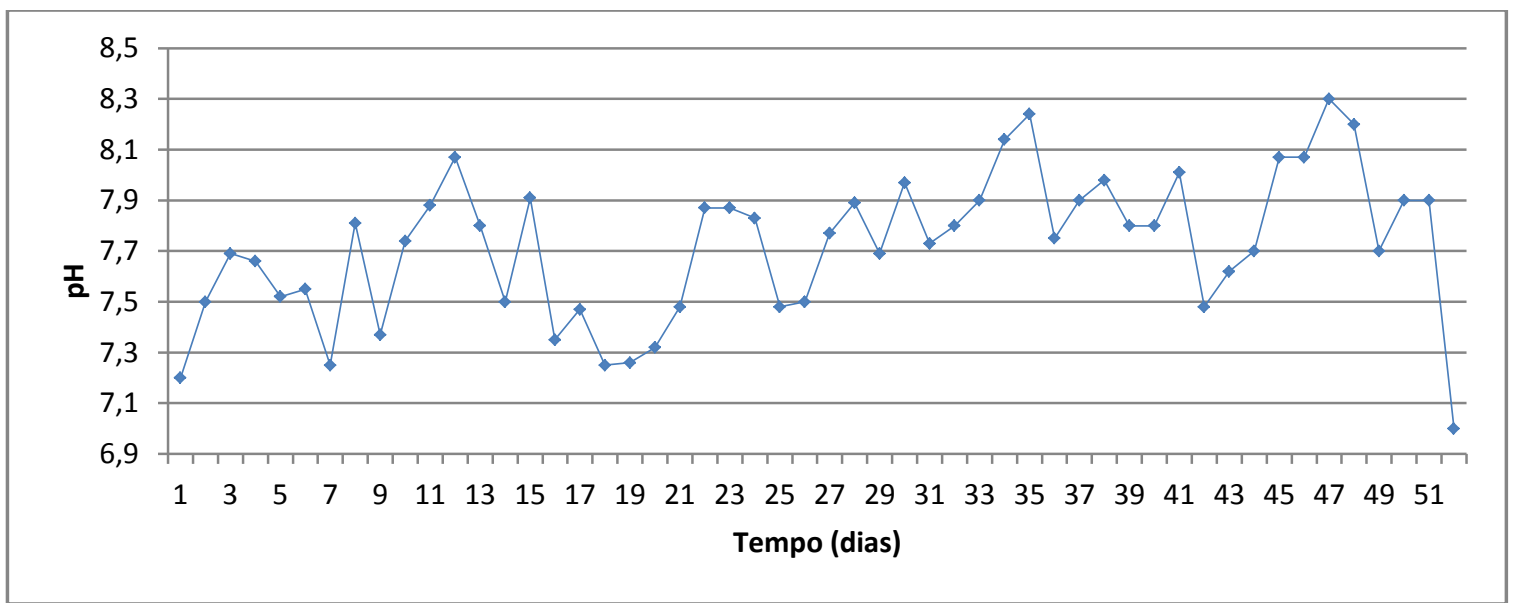

Fonte: Autores.

\subsubsection{Oxigênio Dissolvido}

Os valores das concentrações de OD nos primeiros nove dias de experimento foram medidas diretamente do tanque de cultivo e estão apresentados na Figura 11. 
Figura 11: Valores de OD no tanque de cultivo nos primeiros 9 dias

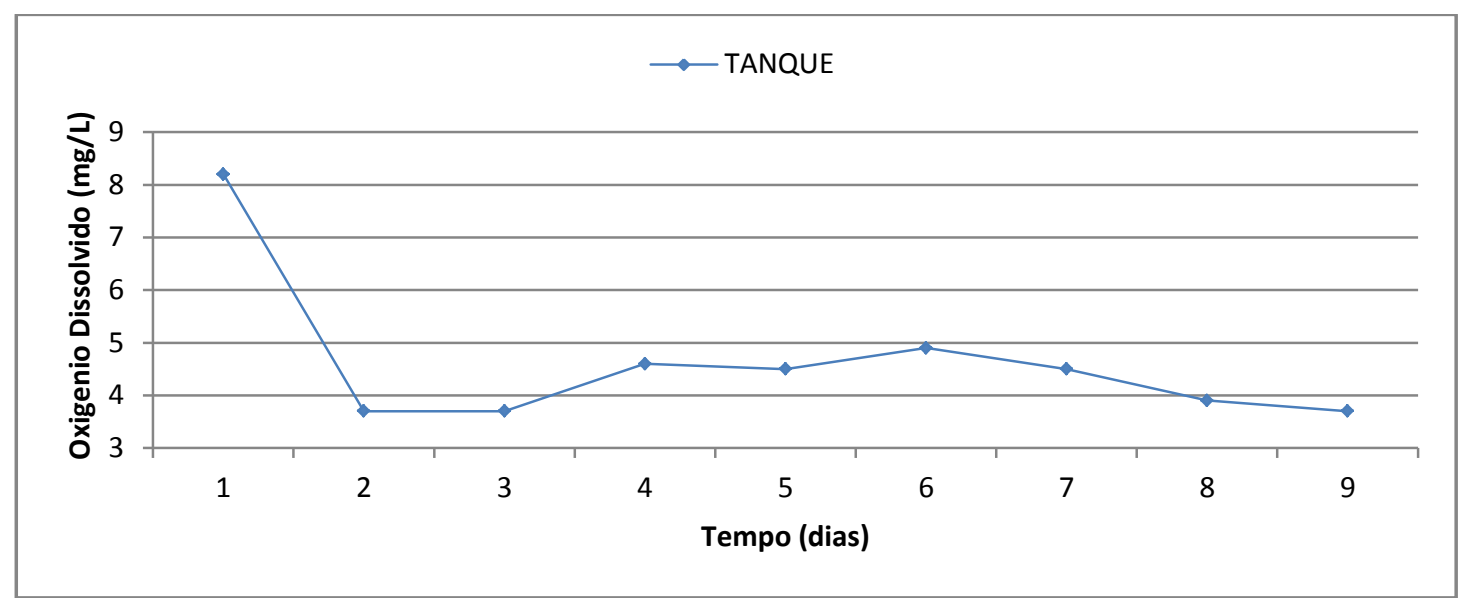

Fonte: Autores.

Após os 9 primeiros dias de experimento, os teores de OD foram obtidos fazendo-se as análises em três pontos: saída do tanque (ST), saída do decantador (SD) e saída do reator (SR). Os valores encontrados estão apresentados na figura 12.

Figura 12: Valores de OD nos três pontos de análises do dia 9 ao 52

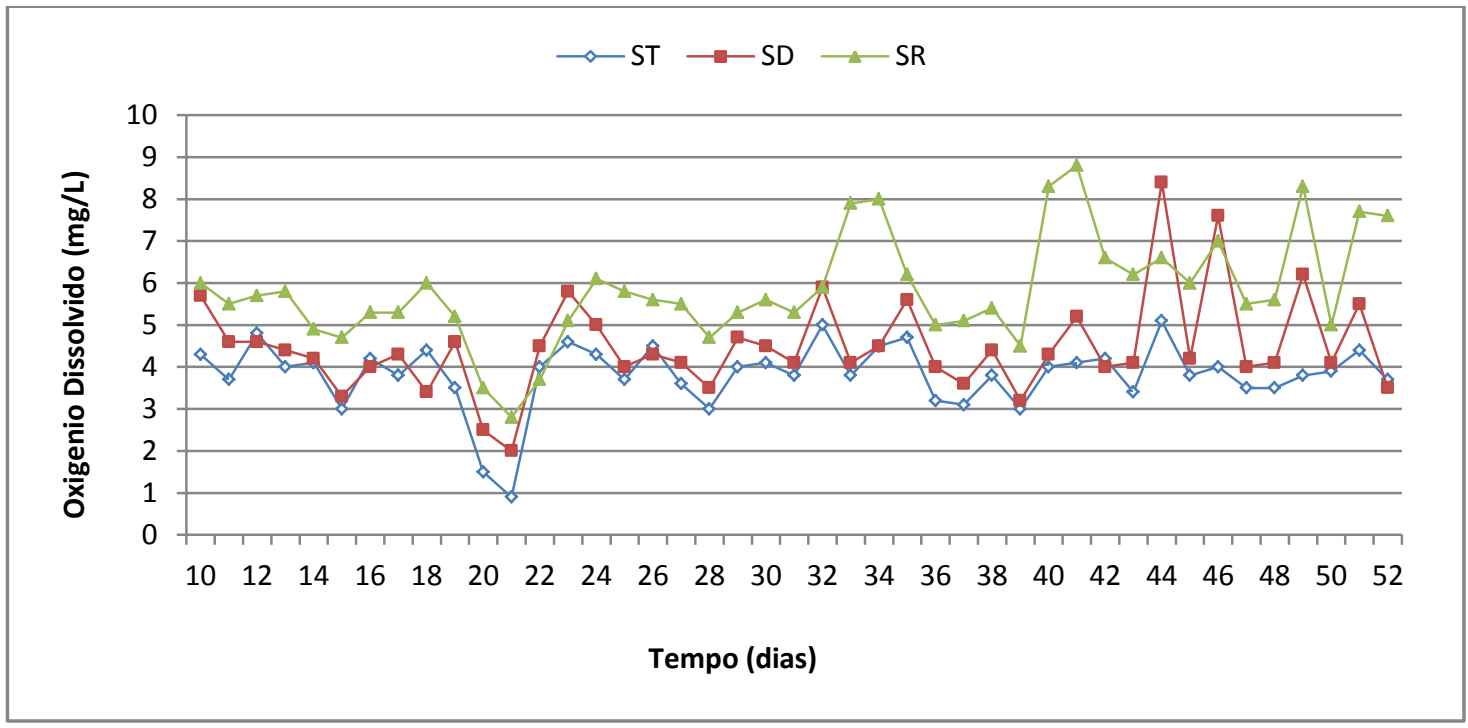

Fonte: Autores.

\section{CONCLUSÕES}

Os resultados obtidos nos testes preliminares demonstraram que a membrana de fibra oca utilizada no teste apresentou resultados satisfatórios em relação à TTO, atingindo valores de até $7,6608 \mathrm{~g} \cdot \mathrm{O}_{2} \cdot \mathrm{m}^{-2} \cdot \mathrm{dia}^{-1}$.

Quando o SRA foi colocado em funcionamento, os valores de pH tiveram uma oscilação baixa, sendo mantido em uma faixa dentro do recomendado para o cultivo de tilápias. Em alguns dias, os valores de temperatura tiveram uma baixa decorrente da queda de temperatura 
ocorrida na cidade. Entretanto, de um modo geral, obteve-se uma temperatura média de $24,27^{\circ} \mathrm{C}$, dentro do intervalo ideal segundo a literatura.

Com os valores de $\mathrm{OD}$, pôde-se observar que o MABR foi imprescindível para que ocorresse um aumento nos níveis de oxigênio dentro do tanque. Notou-se que o efluente que saía do MABR continha níveis de OD com uma média de $5,88 \mathrm{mg} . \mathrm{L}^{-1}$, o que contribuiu para a sobrevivência dos peixes.

Concluiu-se, ao fim do trabalho, que os valores foram satisfatórios nos testes de aeração da membrana e que alguns parâmetros para sobrevivência básica dos peixes foram mantidos dentro dos limites.

\section{AGRADECIMENTO}

À Coordenação de Aperfeiçoamento de Pessoal de Nível Superior (CAPES) pela concessão da bolsa de mestrado das autoras Juliana Cristina Ribeiro Almeida e Natasha Ulhiana Ferreira Ribeiro, e ao Conselho Nacional de Desenvolvimento Científico e Tecnológico (CNPq) pela concessão da bolsa de mestrado dos autores João Miguel Merces Bega e Amanda de Moraes Ricardi.

\section{REFERÊNCIAS BIBLIOGRÁFICAS}

BLANCHETON, J. P. Developments in recirculation systems for Mediterranean fish species. Aquacultural Engenineering, v. 22, n. 1, p. 17-31, 2000.

FAO - ORGANIZAÇÃO DAS NAÇÕES UNIDAS PARA ALIMENTAÇÃO E AGRICULTURA. FAOSTAT. Brasil: FAO. Relatório sobre o estado da Pesca e Aquicultura á nível mundial, SOFIA (2018). Disponível em: < http://www.fao.org/3/I9540EN/i9540en.pdf>. Acesso em 05 de Agosto de 2019.

KUBITZA, F. Tilápias: Parte I. Panorama da aquicultura. v. 10, n. 59, p. 44-53, 2000.

KUBITZA, F. Tilápia em água salobra e salgada: uma boa alternativa de cultivo para estuários e viveiros litorâneos. Panorama da aquicultura, v. 15, n. 88, p. 14-18, 2005.

MAIGUAL ENRIQUEZ, Y. A. Utilização de reator aeróbio de leito fluidizado com circulação em tubos concêntricos no tratamento de águas residuárias da produção intensiva de tilápia com recirculação da água tratada. 2011. 172f. Dissertação (Mestrado). Universidade Estadual Paulista, Ilha Solteira, 2011.

MASSER, M. P.; RAKOCY, J.; LOSORDO, T. M. Recirculating aquaculture tankproduction systems - Management of Recirculating Systems. Southern Regional AquacultureCenter. Texas. SRAC Publication No. 452 - March 1999. 12p.

SCHULTER, E. P.; VIEIRA FILHO, J. E. R. Evolução da piscicultura no Brasil: Diagnóstico e desenvolvimento da cadeia produtiva de Tilápia. Texto para discussão / Instituto de Pesquisa Econômica Aplicada- Brasília. Rio de Janeiro: Ipea, 42 p., 2017.

SIQUEIRA, T.V. Aquicultura: a nova fronteira para aumentar a produção mundial de alimentos de forma sustentável. Boletim regional urbano e ambiental (IPEA) p. 54, 2017.

SILVA, T. S. Efeitos da velocidade de recirculação em reator de biofilme aerado em membranas em batelada sequencial na remoção de nutrientes e carbono orgânico de esgoto doméstico. 2017. 104f. Dissertação (Mestrado). Curso Engenharia Civil, Universidade Estadual Paulista, Ilha Solteira, 2017. 

desempenho, princípios de engenharia, aumento de escala e requisitos de desenvolvimento.

Environmental Science Technology, v. 42, p. 1833-1844, 2008. 